\title{
Analysis of COVID-19 infection spread in Japan based on stochastic transition model
}

\author{
Kenji Karako ${ }^{1}$, Peipei Song ${ }^{2, *}$, Yu Chen ${ }^{1, *}$, Wei Tang ${ }^{3}$ \\ ${ }^{1}$ Department of Human and Engineered Environmental Studies, Graduate School of Frontier Sciences, The University of Tokyo, Chiba, Japan; \\ ${ }^{2}$ Institute for Global Health Policy Research, Bureau of International Health Cooperation, National Center for Global Health and Medicine, \\ Tokyo, Japan; \\ ${ }^{3}$ International Health Care Center, National Center for Global Health and Medicine, Tokyo, Japan.
}

SUMMARY To assess the effectiveness of response strategies of avoiding large gatherings or crowded areas and to predict the spread of COVID-19 infections in Japan, we developed a stochastic transmission model by extending the Susceptible-Infected-Removed (SIR) epidemiological model with an additional modeling of the individual action on whether to stay away from the crowded areas. The population were divided into three compartments: Susceptible, Infected, Removed. Susceptible transitions to Infected every hour with a probability determined by the ratio of Infected and the congestion of area. The total area consists of three zones crowded zone, mid zone and uncrowded zone, with different infection probabilities characterized by the number of people gathered there. The time for each people to spend in the crowded zone is curtailed by $0,2,4,6,7$, and 8 hours, and the time spent in mid zone is extended accordingly. This simulation showed that the number of Infected and Removed will increase rapidly if there is no reduction of the time spent in crowded zone. On the other hand, the stagnant growth of Infected can be observed when the time spent in the crowded zone is reduced to 4 hours, and the growth number of Infected will decrease and the spread of the infection will subside gradually if the time spent in the crowded zone is further cut to 2 hours. In conclusions The infection spread in Japan will be gradually contained by reducing the time spent in the crowded zone to less than 4 hours.

Keywords coronavirus disease 2019 (COVID-19), Japan, transmission, infection, modeling, SusceptibleInfected-Removed (SIR)

\section{Introduction}

In December 2019 an outbreak of coronavirus disease 2019 (COVID-19) occurred in Wuhan (1). At present, some countries are facing on a crisis of outbreak and trying to prevent the spread of 2019-nCoV infections (27). Japan is also one of them. As of February 29, 2020, in addition to the confirmed 619 cases (passengers and crew members) from a virus-hit cruise ship docked in Yokohama near Tokyo and 15 cases returning to Japan by charter flight from Wuhan, 215 locally transmitted cases have been confirmed to have the 2019-nCoV infection in Japan $(8,9)$.

To contain the outbreak, the government of Japan shifted the response strategy from "prevention of domestic invasion" to "prevention infection spread". On February 25, the Japanese government officially released the latest countermeasures for COVID-19 infections (10). The Japanese government asked the public not to flock to hospitals without prior consultation, with the purpose of reducing the risk of exposure to cross-contamination at medical facilities or on the way. To stay away from large gatherings or crowded areas, the Japanese government promoted the adoption of telecommuting, encourage staggering commuting hours and ensure that workers with cold-like symptoms can take sick leave. In addition, on February 27, the government requested all elementary, junior high and high schools in Japan to close from March 2 until the end of a spring break through early April 2020 (11).

It is unknown whether these strategies have had an impact, and how long they should avoid large gatherings or crowded areas. To assess the effectiveness of these response strategies and to predict the spread of COVID-19 infections in Japan, we thus developed a stochastic transmission model by extending the Susceptible-Infected-Removed (SIR) epidemiological model with an additional modeling of the individual 
action on whether to stay away from the crowded areas.

\section{Methods}

\subsection{Model structure}

We implemented a stochastic state transition model with reference to SIR model $(12,13)$ dividing population into three compartments: Susceptible, Infected, Removed. Flow of the state transition is shown in Figure 1. Susceptible transitions to Infected every hour with a probability determined by the ratio of Infected and the congestion of area. The total area consists of three zones crowded zone, mid zone and uncrowded zone, with different infection probabilities characterized by the number of people gathered there. The probability of infection at each hour is decided as:

$$
\begin{aligned}
P_{\text {infection }}(t, d) & =P_{\text {zone }}(t) R_{\text {Infected }}(d) \\
R_{\text {Infected }}(d) & =\frac{N_{\text {Infected }}(d)}{N_{\text {Infected }}(d)+N_{\text {Susceptible }}(d)}
\end{aligned}
$$

Where $P_{\text {zone }}(t)$ denotes the infection probability at the zone where Susceptible exist at time $(t), R_{\text {Infected }}$ (d) denotes the ratio of Infected determined by the number of Infected and Susceptible at day $(d)$. In addition, Infected transitions to Removed every day by segregation with a certain probability: $P_{\text {Removed }}$ and hence Removed will not infect others any more.

\subsection{Estimation of model parameters}

In order to apply our model to Japan's situation, we need to estimate the parameters $P_{\text {crowded zone }}, P_{\text {mid zone }}, P_{\text {uncrowded }}$ zone and $P_{\text {Removed }}$. To estimate the $P_{z o n e}$, we reference the infected ratio of the cases at diamond princess and returning to Japan by charter flight from Wuhan. In the case of diamond princess, there were 3,711 people on board, of which 619 were infected, and the ratio of infected was $17 \%$ (9). Out of 829 returnees from Wuhan, 15 were infected and the ratio of infected was $1.8 \%$ (14). The ratio of infected in spatially isolated ship were 10 times greater than those in returnees from potentially dangerous area. Crowded zone is considered as not an isolated space like diamond princess but an area with a high probability of infection, thus we set the infection probability at crowded zone as $1.8 \%$. Also, according to the difference between diamond princess and returnees from Wuhan, we set the probability at mid zone and uncrowded zone as $0.18 \%$ and $0.018 \%$. As of February 25,140 out of 1,017 people were detected as infected by PCR test in Japan (7). According to detection rate, we set $P_{\text {Removed }}$ as $14 \%$.

To set action policy on where to spend, we refer to the average Japanese behavior obtained from the basic survey on social life (15). The total time for work, meals, study, and travel was 467 minutes. According

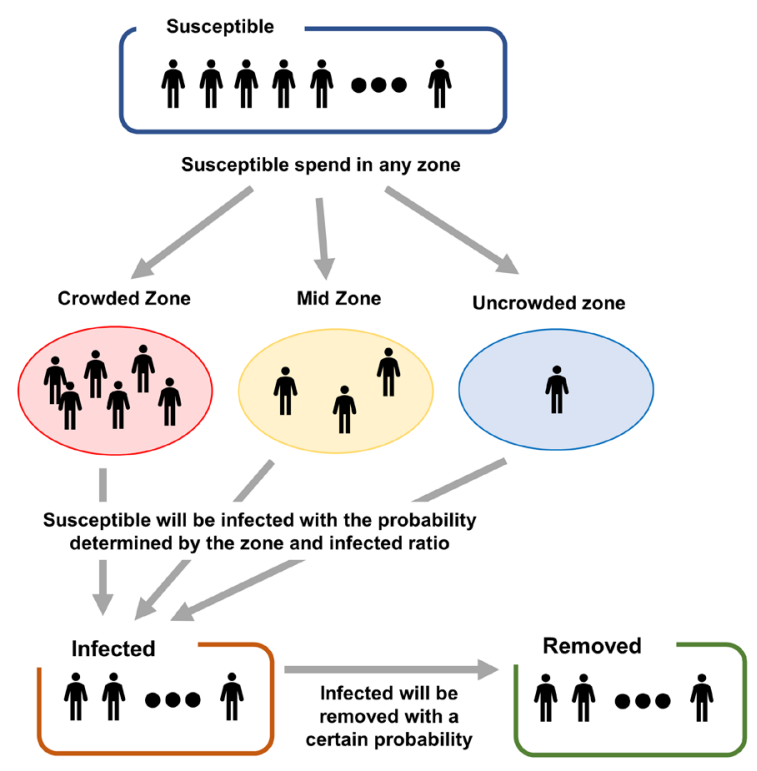

Figure 1. The flow of transition between three compartments.

the time spending at places where many people gather, we set 8 hours as the time in crowded zone per day. And also, we set 8 hours as the time in mid and uncrowded zone per day.

The result using above settings and initial Susceptible:120,000,000 and initial Infected:10 is shown in Figure 2. The number of Removed was 65 at $30^{\text {th }}$ day. The number does not match the situation in Japan, where the infection was confirmed for the first time on January 14 and reached 165 on February 25. Therefore, we changed the parameters as $P_{\text {crowded zone }}: 2 \%, P_{\text {mid zone }}: 0.2 \%$, $P_{\text {uncrowded zone }}: 0.02 \%$ and $P_{\text {Removed }}: 10 \%$, and simulate the infection spread (Figure 3). The number of Removed reached 159 at $30^{\text {th }}$ day and it was close to the situation in Japan.

\section{Results and Discussion}

Prediction of the spread in Japan Reflecting the Japanese strategy to prevent outbreaks and control the spread of infection, we simulated infection spread using some scenarios with different hour in crowded zone. Assuming Japan response strategy "prevention infection spread" reduces time in crowded zone, we simulate the spread continuing 30 days of Figure 3 with same probabilities.

As a parameter study of the model, the time for each people to spend in the crowded zone is curtailed by 0 , $2,4,6,7$, and 8 hours, and the time spent in mid zone is extended accordingly. These results were shown in Figure 4.

Results of simulation showed that $i$ ) the number of Infected and Removed will increase rapidly if there is no reduction of the time spent in crowded zone; ii) the stagnant growth of Infected can be observed when the time spent in the crowded zone is reduced to 4 hours; 


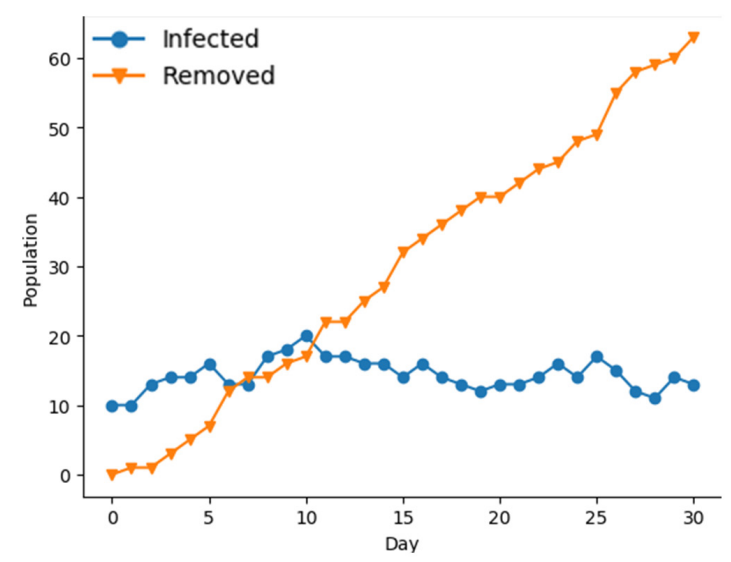

Figure 2. Infected and Removed population transition simulated with $P_{\text {crowded zone }}: 1.8 \%, P_{\text {mid zone }}: 0.18 \%, P_{\text {uncrowded zone }}: 0.018 \%$, and $P_{\text {Removed }}: 14 \%$.
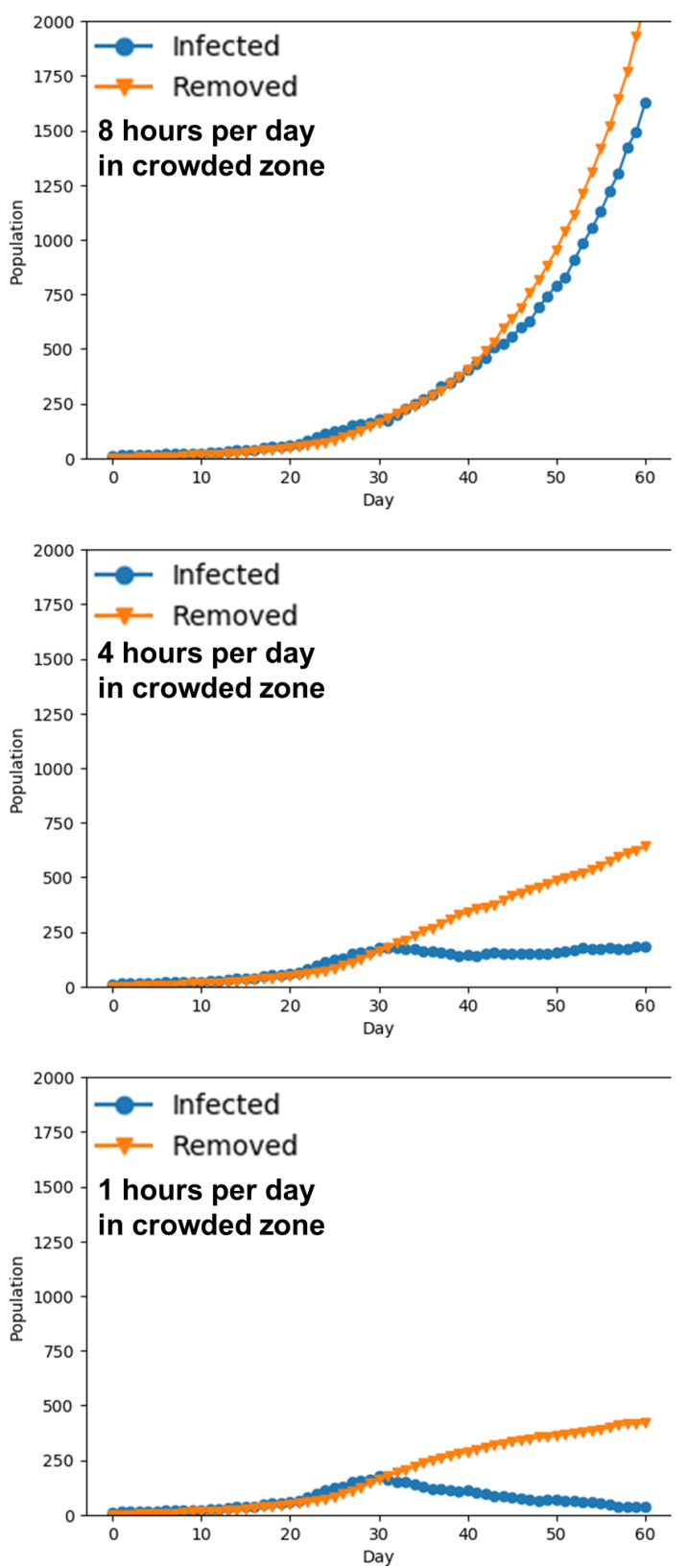

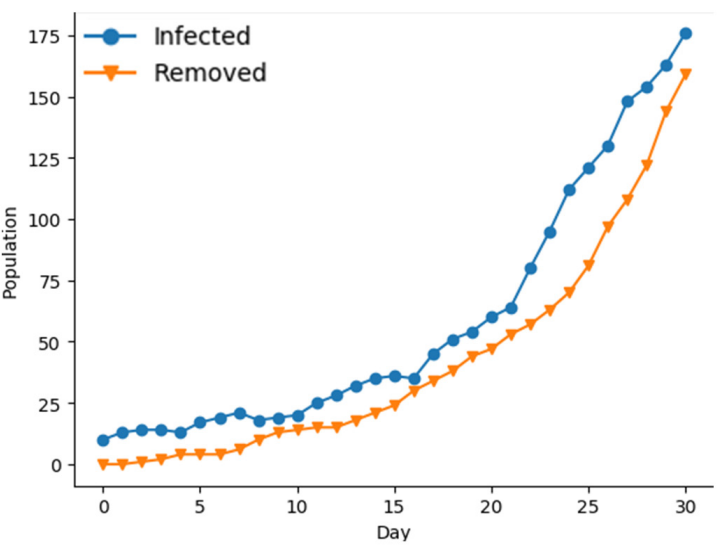

Figure 3. Infected and Removed population transition simulated with $\boldsymbol{P}_{\text {crowded zone: }}: 2 \%, \boldsymbol{P}_{\text {mid zone }}: 0.2 \%, \boldsymbol{P}_{\text {uncrowded zone }}: 0.02 \%$, and $\boldsymbol{P}_{\text {Removed }}$ : $10 \%$.
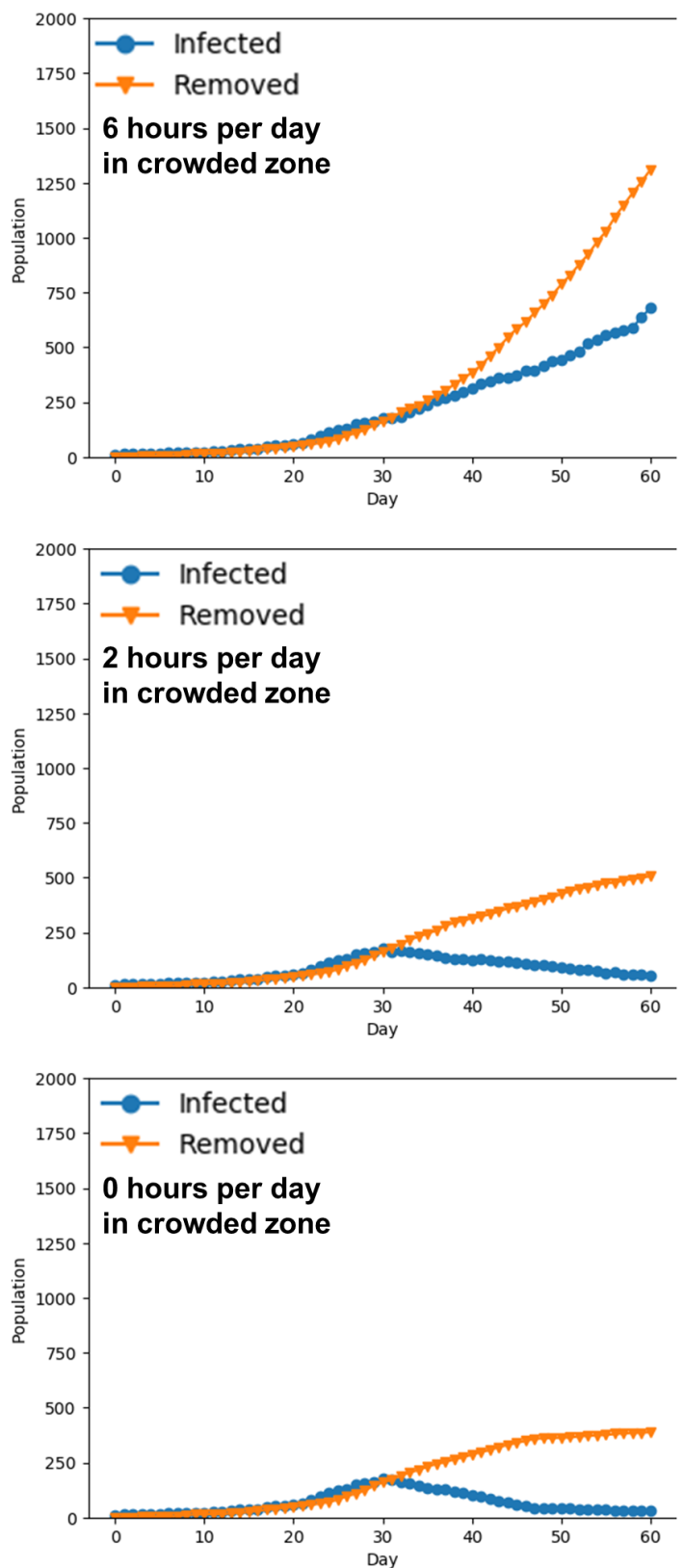

Figure 4. Infected and Removed population transition simulated with changing the time spending in crowded zone after 30 days. 
and $i i i)$ the growth number of Infected will decrease and the spread of the infection will subside gradually if the time spent in the crowded zone is further cut to 2 hours.

As can be seen in Figure 4, the infection spread in Japan will be gradually contained by reducing the time spent in the crowded zone to less than 4 hours. Otherwise, the outbreak of infection in Japan will remain.

The Japanese government has already advice all people to stay away from large gatherings or crowded areas. In addition, the government requested all elementary, junior high and high schools in Japan to close from March 2 and promoted the adoption of telecommuting and encourage staggering commuting hours. Through these policies, minimizing exposure risks of the public $(16,17)$ will be effective and feasible measure to contain mass COVID-19 infection in Japan.

On the other hand, if the time in crowded zone does not decrease enough by these policies, there is a way to control the spread by increasing the probability of Removed.

Although there are some voices in the public questioning that the number of people tested every day is too small, from the perspective of policies to prevent infectious diseases, performing PCR test on everyone is not an effective measure against this virus because of the risk of exposure to cross-contamination at medical facilities or on the way as well as the low positive rate, high false-negative rate, low patient viral load, or improper clinical sampling, etc (18-21). However, if exposure to cross-contamination can be prevented, increasing the number of tests will be a measure to control the spread of infection.

In conclusion, we developed a COVID-19 spread prediction model analysis with the stochastic transition model using existence ratio of influencer and the areas characterized by the number of people gathered. Results of simulation showed that preventing mass COVID-19 infection and control the spread of infection need to reduce the time spent in the crowded zone to less than 4 hours. Everyone should follow the Japan response strategies and avoid crowded areas as possible.

\section{References}

1. Li Q, Guan X, Wu P, et al. Early Transmission Dynamics in Wuhan, China, of Novel CoronavirusInfected Pneumonia. N Engl J Med. 2020; doi: 10.1056/ NEJMoa2001316.

2. World Health Organization. Coronavirus disease (COVID-2019) situation reports. https://www.who.int/ emergencies/diseases/novel-coronavirus-2019/situationreports (accessed March 11, 2020).

3. Guan WJ, Ni ZY, Hu Y, et al. Clinical Characteristics of Coronavirus Disease 2019 in China. N Engl J Med. 2020; doi: 10.1056/NEJMoa2002032.

4. Gilbert M, Pullano G, Pinotti F, Valdano E, Poletto C, Boëlle PY, D'Ortenzio E, Yazdanpanah Y, Eholie SP, Altmann M, Gutierrez B, Kraemer MUG, Colizza V.
Preparedness and vulnerability of African countries against importations of COVID-19: a modelling study. Lancet. 2020; 395:871-877.

5. Hoehl S, Berger A, Kortenbusch M, et al. Evidence of SARS-CoV-2 Infection in Returning Travelers from Wuhan, China. N Engl J Med. 2020; doi: 10.1056/ NEJMc2001899.

6. Adalja AA, Toner E, Inglesby TV. Priorities for the US Health Community Responding to COVID-19. JAMA. 2020; doi: 10.1001/jama.2020.3413.

7. Porcheddu R, Serra C, Kelvin D, Kelvin N, Rubino S. Similarity in case fatality rates (CFR) of COVID-19/ SARS-COV-2 in Italy and China. J Infect Dev Ctries. 2020; 14:125-128.

8. Ministry of Health, Labour and Welfare, Japan. About New Coronavirus Infections. February 29, 2020. https://www. mhlw.go.jp/stf/seisakunitsuite/bunya/0000164708 00001. html (accessed March 11, 2020).

9. National Institute of Infectious Diseases. Field Briefing: Diamond Princess COVID-19 Cases, 20 Feb Update . https://www.niid.go.jp/niid/en/2019-ncov-e/9417-covid$d p-f e-02 . h t m l$ (accessed March 11, 2020).

10. Ministry of Health, Labour and Welfare, Japan. Basic policy for countermeasures against SARS-Cov-2 infection. February 25, 2020. https://www.mhlw.go.jp/ content/10900000/000599698.pdf (accessed March 11, 2020).

11. NHK World-Japan. Abe to ask all schools to close during March. February 27, 2020. https://www3.nhk.or.jp/ nhkworld/en/news/20200227_47 (accessed March 11, 2020).

12. Kermack WO, McKendrick AG. A contribution to the mathematical theory of epidemics. Proceedings of the royal society of london. Series A. 1927; 115:700-721.

13. Britton T. Stochastic epidemic models: a survey. Math Biosci. 2009; 225:24-35.

14. Ministry of Health, Labour and Welfare, Japan. About the Present Situation of New Coronavirus Infections and Correspondence of Ministry of Health, Labor and Welfare. February 25, 2020. https://www.mhlw.go.jp/stf/ newpage_09747.html (accessed March 11, 2020).

15. Statistics Bureau of Japan. Basic Survey on Social Life in 2016. September 15, 2017. https://www.stat.go.jp/data/ shakai/2016/pdf/gaiyou3.pdf (accessed March 11, 2020).

16. Hellewell J, Abbott S, Gimma A, Bosse NI, Jarvis CI, Russell TW, Munday JD, Kucharski AJ, Edmunds WJ; Centre for the Mathematical Modelling of Infectious Diseases COVID-19 Working Group, Funk S, Eggo RM. Feasibility of controlling COVID-19 outbreaks by isolation of cases and contacts. Lancet Glob Health. 2020; pii: S2214-109X(20)30074-7. doi: 10.1016/S2214109X(20)30074-7.

17. Chinazzi M, Davis JT, Ajelli M, et al. The effect of travel restrictions on the spread of the 2019 novel coronavirus (COVID-19) outbreak. Science. 2020; pii: eaba9757. doi: 10.1126/science.aba9757.

18. Tang A, Tong ZD, Wang HL, Dai YX, Li KF, Liu JN, Wu WJ, Yuan C, Yu ML, Li P, Yan JB. Detection of Novel Coronavirus by RT-PCR in Stool Specimen from Asymptomatic Child, China. Emerg Infect Dis. 2020; 26: doi: 10.3201/eid2606.200301.

19. Fang Y, Zhang H, Xie J, Lin M, Ying L, Pang P, Ji W. Sensitivity of Chest CT for COVID-19: Comparison to RT-PCR. Radiology. 2020; doi: 10.1148/ radiol.2020200432. 
20. Ai T, Yang Z, Hou H, Zhan C, Chen C, Lv W, Tao Q, Sun Z, Xia L. Correlation of Chest CT and RT-PCR Testing in Coronavirus Disease 2019 (COVID-19) in China: A Report of 1014 Cases. Radiology. 2020; doi: 10.1148/ radiol.2020200642

21. World Health Organization. Report of the WHO-China Joint Mission on Coronavirus Disease 2019 (COVID-19). https://www.who.int/docs/default-source/coronaviruse/ who-china-joint-mission-on-covid-19-final-re (accessed March 11, 2020).

Received March 14, 2020; Revised March 18, 2020; Accepted March 18, 2020.
*Address correspondence to:

Peipei Song, Institute for Global Health Policy Research, Bureau of International Health Cooperation, National Center for Global Health and Medicine, Tokyo, Japan.

E-mail: ppsong-tky@umin.ac.jp

Yu Chen, Department of Human and Engineered Environmental Studies, Graduate School of Frontier Sciences, The University of Tokyo, Chiba, Japan.

E-mail: chen@edu.k.u-tokyo.ac.jp

Released online in J-STAGE as advance publication March 19, 2020. 